Research paper

\title{
PTHrP in differentiating human mesenchymal stem cells: Transcript isoform expression, promoter methylation, and protein accumulation
}

\author{
Q3 Alessandra Longo ${ }^{a}$, Mariangela Librizzi ${ }^{a}$, Flores Naselli ${ }^{a}$, Fabio Caradonna ${ }^{a}$, \\ Edda Tobiasch ${ }^{\mathrm{b}}$, Claudio Luparello ${ }^{\mathrm{a}, *}$ \\ a Dipartimento STEBICEF, Università di Palermo, Palermo, Italy \\ Q1 ${ }^{\mathrm{b}}$ Department of Natural Sciences, Hochschule Bonn-Rhein-Sieg, Rheinbach (D), Germany
}

\section{A R T I C L E I N F O}

Article history:

Received 10 May 2013

Accepted 18 June 2013

Available online $\mathrm{xxx}$

\section{Keywords:}

PTHrP

Mesenchymal stem cells

Osteogenesis

Adipogenesis

Gene expression

Promoter methylation

\begin{abstract}
A B S T R A C T
Human PTHrP gene displays a complex organization with nine exons producing diverse mRNA variants due to alternative splicing at $5^{\prime}$ and $3^{\prime}$ ends and the existence of three different transcriptional promoters (P1, P2 and P3), two of which (P2 and P3) contain CpG islands. It is known that the expression of PTHrP isoforms may be differentially regulated in a developmental stage- and tissue-specific manner. To search for novel molecular markers of stemness/differentiation, here we have examined isoform expression in fat-derived mesenchymal stem cells both maintained in stem conditions and induced toward adipo- and osteogenesis. In addition, the expression of the splicing isoforms derived from P2 and P3 promoters was correlated to the state of methylation of the latter. Moreover, we also performed a quantitative evaluation of intracellular and secreted PTHrP protein product in undifferentiated stem cells and in parallel cultures at various differentiation stages. The data obtained indicate that from the stemness condition to that of osteo- and adipo-genic differentiated cells, the expression of isoforms becomes increasingly selective, thereby being a potential gene signature for the monitoring of cell stem or committed/differentiating state and that the switching-off of PTHrP isoform expression is mostly promoter methylation-dependent. Moreover, PTHrP intracellular retention is down-regulated in osteo-differentiating cells whereas the secretion of the protein in the extracellular medium is up-regulated with respect to stem cells, thereby suggesting that these variations of the intracellular and extracellular levels of PTHrP could potentially be enclosed in the list of the available protein signature of osteogenic differentiation.
\end{abstract}

(c) 2013 Published by Elsevier Masson SAS.

\section{Introduction}

Mesenchymal stem cells (MSC) are a heterogeneous population of stem cells with capacity to differentiate into mesodermal cell lineages including osteocytes, adipocytes and chondrocytes, and non-mesodermal cell lineages, e.g. neurons, insulin-producing and

Abbreviations: MSC, mesenchymal stem cells; BMP, bone morphogenetic protein; PTHrP, parathyroid hormone-related protein; FCS, fetal calf serum; PMSF, phenylmethylsulfonyl fluoride; EDTA, ethylenediaminetetraacetic acid; dNTPs, deoxynucleotide triphosphates; SM, semi-quantitative multiplex; MSRE, methylation-sensitive restriction endonuclease; PCR, polymerase chain reaction; MICE, microtiter immunocytochemical ELISA assay; D-PBS, Dulbecco's phosphate buffered saline; TMB, 3,3',5,5'-tetramethylbenzidine; SDS, sodium dodecyl sulfate; TBS-T, Tris-buffered saline-Tween 20; BCIP, 5-bromo-4-chloro-3'-indolyphosphate; NBT, nitro-blue tetrazolium; s.e.m., standard error of the mean.

* Corresponding author. Dipartimento di Scienze e Tecnologie Biologiche, Chimiche e Farmaceutiche (STEBICEF), University of Palermo, Edificio 16, Viale delle Scienze, 90128 Palermo, Italy. Tel.: +39 09123897405; fax: +39 0916577210.

E-mail addresses: claudio.luparello@unipa.it, clupar@tin.it (C. Luparello). epithelial cells $[1,2]$. The use of somatic stem cells, particularly due to their ability to be easily expanded ex vivo and the reduced involvement of ethical and immunogenic issues, is one of the most promising areas of research about tissue regeneration. Apart from bone marrow and umbilical cord blood, adipose tissue has proven to serve as source of adult stem cells with multipotent properties [3-5], which can be harvested in relative abundance by modern liposuction techniques under local anesthesia [6].

The multipotentiality of MSC is closely linked to their therapeutic interest, in that these cells might find utilization in cell therapy and tissue engineering. As an example, a better comprehension of the cellular and molecular mechanisms of differentiation of adipose and bone tissue would be respectively useful to develop innovative strategies to prevent and treat the obesity, that results from excess white adipose tissue, and its related disorders, and to improve bone regeneration thus by-passing the numerous problems associated with reconstructive orthopedic surgery [7].

The different phases of the adipo- and osteo-differentiation processes have been examined thoroughly and specific signaling 
pathways involved, such as those of Wnt and BMP, as well as transcription factor families playing a central role in the of adipogenesis (e.g. Runx2 and Osterix) and osteogenesis (e.g. CCAAT/ enhancer binding proteins, peroxisome proliferator-activated receptors and sterol regulatory element binding proteins) have been identified [e.g. Refs. [8-11]]. However, a complete scenario of these differentiation events is far to be reached, and specific markers for the characterization of defined differentiation steps, especially the earlier ones, are still lacking. In light of the interest on novel molecular signatures that can be utilized to monitor the stem, committed or pre-differentiated state of cells, here we focused our interest on some aspects of the expression and production of parathyroid hormone-related protein (PTHrP), a well-known regulator of proliferation, differentiation and apoptosis of normal and neoplastic cells [e.g. Refs. [12-17]], whose activity on the modulation of chondrogenic differentiation by adult stem cells has been reported [e.g. Refs. [18,19]].

PTHrP is the product of a single gene which is located in the short arm of human chromosome 12. It spans over 15 kbases of DNA and displays a complex organization with nine exons undergoing alternative splicing and producing diverse mRNA variants. Three PTHrP transcriptional start sites have been reported, i.e. two TATA box promoters, $5^{\prime}$ to exons I and IV (named P1 and P3, respectively) and a GC-rich promoter, $5^{\prime}$ to exon III (named P2). The P2 promoter contains a high percentage of $\mathrm{CpG}$ dinucleotides in an island upstream of exon III and CpG sites are present also in P3 promoter $[20,21]$. As summarized in Table 1, different splicing events are observed at the $5^{\prime}$ terminus according to the promoter chosen to initiate transcription. In addition, alternative splicing of exons at $3^{\prime}$ end generates multiple PTHrP transcripts which encode three isoforms of the mature peptide of 139, 173 and 141 amino acids which differ in their C-terminal regions. These protein isoforms are then subjected to post-translational endoproteolysis into smaller bioactive forms which have been either immunodetected or isolated from conditioned media of normal and neoplastic cells and are endowed with individual functions [22-24]. The presence of three promoters and $5^{\prime}$ and $3^{\prime}$ terminus alternative splicing indicates that the expression of PTHrP isoforms may be differentially regulated in a developmental stage- and tissue-specific manner [25-30].

Even though the PTHrP expression and biological role have been extensively studied in many cell types, the expression of its transcript variants by MSC is so far poorly understood. Therefore, in order to extend the knowledge of the biological significance of PTHrP differential regulation and to investigate whether selected PTHrP isoforms may represent novel molecular signatures associated to stemness/differentiation state, the main aim of this study was to examine the time-course expression of the splicing isoforms of PTHrP in fat-derived MSC both maintained in stem conditions and induced toward adipo- and osteo-genesis. In addition, the expression of the splicing isoforms derived from P2 and P3 promoters by MSC, was correlated to the state of methylation of these promoters that contain $\mathrm{CpG}$ sites. A further set of analyses was also performed for the quantitative evaluation of intracellular and secreted PTHrP protein product in undifferentiated MSC and in parallel cultures at various differentiation stages, in order to evaluate whether modifications of protein amounts, if any, can be related to cell stem or differentiating state.

\section{Materials and methods}

\subsection{Cell cultures and differentiation induction}

The MSC used in the present investigation were cultured and differentiated as described in Ref. [31]. In brief, MSC were obtained from lipoaspirates of three different healthy donors and cultured in DMEM supplemented with $10 \%$ fetal calf serum (FCS), $2 \mathrm{mM} \mathrm{L-}$ glutamine, and $1 \%$ penicillin/streptomycin at $37{ }^{\circ} \mathrm{C}$ and $5 \% \mathrm{CO}_{2}$. Adipogenic and osteogenic differentiation were checked via cytochemical staining and molecular marker expression after four weeks of culture, as described. At the end of each week, aliquots of undifferentiated and differentiating cells were processed for DNA and RNA isolation and for MICE assays (see following paragraphs), whereas the conditioned media were supplemented with protease inhibitors (100 $\mu \mathrm{M}$ leupeptin, $50 \mathrm{mM}$ PMSF, $15 \mu \mathrm{M}$ aprotinin and $5 \mathrm{mM}$ EDTA) and stored at $-80^{\circ} \mathrm{C}$.

\subsection{Genomic DNA isolation}

Isolation of genomic DNA from undifferentiated and differentiating cells was carried out with the PureLink Genomic DNA Kit (Invitrogen, Carlsbad/CA, USA). The obtained DNA was quantified by $0.8 \%$ agarose gel electrophoresis run in parallel with known concentrations of salmon sperm DNA (EuroBio, Courtaboeuf, F) and subsequent comparison of the intensities of the obtained bands.

\subsection{RNA extraction and reverse transcription}

Isolation of total RNA from undifferentiated and differentiating cells was carried out with Tri Reagent (Sigma, St. Louis, MO/USA). Before the reverse transcription, the total RNA was treated with RQ1 RNase-free DNase (Promega, Madison, WI/USA) and its quality and integrity checked through agarose gel electrophoresis in denaturing conditions. The cDNAs were synthesized using SuperScript II reverse transcriptase (Invitrogen) in the presence of $100 \mathrm{ng}$ random 6-mer primers (Sigma), 50 U RNase inhibitor (Promega) and $0.5 \mathrm{mM}$ each of dNTPs; reverse transcription was carried out for 60 min at $42{ }^{\circ} \mathrm{C}$, followed by treatment with $2 \mathrm{U}$ RNase H (USB, Cleveland, OH/USA) for $20 \mathrm{~min}$ at $37^{\circ} \mathrm{C}$.

\subsection{Semi-quantitative multiplex (SM)- and methylation-sensitive restriction endonuclease (MSRE)-PCR amplification}

SM-PCR was performed using $1 \mathrm{U}$ RedTaq DNA polymerase (Sigma) $/ \mu \mathrm{l}, 200 \mu \mathrm{M}$ each of dNTPs, $1 \mu \mathrm{l}$ of the cDNA template obtained from total RNA and $2.5 \mu \mathrm{M}$ of the PTHrP splicing isoformand 18 S-specific primers reported by Luparello et al. [28,29,32]. Preliminary assays were performed to determine the number of cycles to keep amplification within the exponential phase and permit sufficient visualization of the PCR product. The cycle profile

Table 1

PTHrP mRNA variants produced by alternative splicing at the $5^{\prime}$ and $3^{\prime}$ ends.

\begin{tabular}{|c|c|c|c|c|c|}
\hline $\begin{array}{l}\text { Promoter } \\
\text { P1 }\end{array}$ & \multirow{2}{*}{$\begin{array}{l}\text { Exons included at the } 5^{\prime} \text { end } \\
\text { I-II-III } \\
\text { I-III } \\
\text { I }\end{array}$} & \multirow{2}{*}{$\begin{array}{l}\text { Ever-present exons } \\
\mathrm{V}-\mathrm{VI}\end{array}$} & \multicolumn{3}{|c|}{ Exons included at the $3^{\prime}$ end $\rightarrow$ protein isoform } \\
\hline P1 & & & $\mathrm{VII} \rightarrow 139$ aa & VIII $\rightarrow 173$ aa & IX $\rightarrow 141$ aa \\
\hline $\mathrm{P} 2$ & III & $\mathrm{V}-\mathrm{VI}$ & VII $\rightarrow 139$ aa & VIII $\rightarrow 173$ aa & $\mathrm{IX} \rightarrow 141$ aa \\
\hline P3 & IV & $\mathrm{V}-\mathrm{VI}$ & VII $\rightarrow 139$ aa & VIII $\rightarrow 173$ aa & $\mathrm{IX} \rightarrow 141$ aa \\
\hline
\end{tabular}


chosen was $94^{\circ} \mathrm{C}$ for $2 \mathrm{~min}$, followed by a product-specific number of cycles of $94^{\circ} \mathrm{C}$ for $1 \mathrm{~min}$, the appropriate annealing temperature for $1 \mathrm{~min}$, and $72{ }^{\circ} \mathrm{C}$ for $1 \mathrm{~min}$ ( 5 min during the last cycle). PCR products were analyzed by $2 \%$ agarose gel electrophoresis, visualized by Gel Red staining (Biotium, Hayward, CA/USA) under UV light, and the image captured in a digital support. The intensities of the bands of interest, evaluated with SigmaScan software (SPSS, USA), were normalized for those of $18 \mathrm{~S}$, and plotted in histograms by SigmaPlot software (SPSS) to examine comparatively the pattern and levels of the gene expression studied. As a positive, parallel amplification of cDNA from 8701-BC breast cancer cells cultured on type I collagen, taken from laboratory stocks, was performed $[28,29]$.

MSRE-PCR was performed as described by Caradonna et al. [33]. Essentially, genomic DNA from undifferentiated and differentiating cells was digested with Hpall and MspI methylation-sensitive restriction endonucleases (MSREs) and amplified by PCR in the presence of primers flanking three regions in PTHrP P2 and one region in PTHrP P3 promoter containing cutting sites for MSREs (Table 2). The reaction mixture contained $300 \mathrm{ng}$ of digested DNA, $0.2 \mu \mathrm{M}$ of each primer and $2.5 \mathrm{U}$ Taq polymerase (Invitrogen); the thermal cycle used was a denaturation step of $94{ }^{\circ} \mathrm{C}$ for $4 \mathrm{~min}$, followed by 30 cycles of $94{ }^{\circ} \mathrm{C}$ for $1 \mathrm{~min}, 60{ }^{\circ} \mathrm{C}$ for $1 \mathrm{~min}$, and $72{ }^{\circ} \mathrm{C}$ for $1 \mathrm{~min}$ ( 5 min during the last cycle). PCR products were analyzed by $12 \%$ acrylamide-bisacrylamide (29:1 ratio) gel electrophoresis and visualized by SYBR Safe staining (Invitrogen) in a ChemiDoc XRS apparatus (Bio-Rad, Hercules, CA/USA) and the image captured in a digital support. The identity of the amplification products was checked by sequencing.

\subsection{Microtiter immunocytochemical ELISA assay (MICE) assay}

MICE assay was performed according to [34]. Essentially, undifferentiated and differentiating cells, cultured in 96-well plates, were fixed with $50 \mu \mathrm{l}$ HistoCHOICE Solution (AMRESCO, Solon, OH/ USA) and permeabilized with $0.05 \%$ saponin (Sigma) in Dulbecco's phosphate buffered saline (D-PBS). After quenching of endogenous peroxidase with $0.03 \% \mathrm{H}_{2} \mathrm{O}_{2}$ in DPBS and blocking of aspecific sites with $10 \%$ FCS and $0.05 \%$ sodium azide in DPBS, cell preparations were incubated overnight at $4{ }^{\circ} \mathrm{C}$ with the primary antibody (antiPTHLP Ab-2, EMD Millipore, Billerica, MA/USA), raised against the peptide corresponding to residues $34-53$ of human PTHrP and developed in rabbit. After incubation with peroxidase labeled antirabbit secondary antibody, the immunoreactivity was revealed by the addition of $100 \mu \mathrm{l}$ of TMB soluble peroxidase substrate (Thermo Fisher, Rockford, IL/USA) and the absorbance measured at $\lambda=450 \mathrm{~nm}$. After the evaluation of immunoreactivity, cell number was assessed by staining with $0.05 \%$ Coomassie Blue dissolved in $40 \%$ methanol and $10 \%$ acetic acid, solubilization of the dye in $1 \%$ SDS in DPBS, and measurement of the absorbance at $\lambda=650 \mathrm{~nm}$. The MICE index was calculated as the ratio between the absorbance value of immunoreactivity and that of Coomassie Blue labeling.

Table 2

Primers used for amplification of the CpG island-containing sites in PTHrP P2 and P3 promoters.

\begin{tabular}{|c|c|c|c|}
\hline Site & Primers & $5^{\prime}-3^{\prime}$ sequence & Expected size (bp) \\
\hline \multirow[t]{2}{*}{ P2 S-II } & Forward & GGTTCATGTGGGGAACTGG & 215 \\
\hline & Reverse & СССАGTCСТGATCTCCTGG & \\
\hline \multirow[t]{2}{*}{ P2 S-III } & Forward & TGTGGCCTCGTACAAGTCC & 502 \\
\hline & Reverse & CTGGAGGAACTGCCTCAGG & \\
\hline \multirow[t]{2}{*}{ P2 S-IV } & Forward & CAGGAGACTCCAGAGAAGG & 456 \\
\hline & Reverse & CTCTGGACTTGTACGAGGC & \\
\hline \multirow[t]{2}{*}{ P3 } & Forward & AGCTGACTTCAGAGGGGGAA & 370 \\
\hline & Reverse & AGGGCATCTCCCAAGTTGAA & \\
\hline
\end{tabular}

\subsection{Dot blot assay}

Dot blot was performed as described in Ref. [35]. Essentially, aliquots of $200 \mu \mathrm{l}$ of media stored at $-80^{\circ} \mathrm{C}$ with protease inhibitors as mentioned above, derived from undifferentiated and differentiating cells were loaded and adsorbed onto the nitrocellulose membrane. Aspecific sites were blocked by soaking in $5 \%$ bovine serum albumin dissolved in Tris-buffered saline-Tween 20 (TBS-T: $150 \mathrm{mM} \mathrm{NaCl}, 20 \mathrm{mM}$ Tris- $\mathrm{HCl} \mathrm{pH}$ 7.5, 0.05\% Tween20). After incubation with the primary (anti-PTHLP Ab-2, EMD Millipore) and the secondary anti-rabbit antibody conjugated with alkaline phosphatase, the spots were identified by staining the membrane with BCIP/NBT liquid substrate system (Sigma), the image captured in a digital support and spot intensities evaluated with Image J software.

\subsection{Statistical analysis}

The data are presented as mean \pm s.e.m. The experiments were performed at least in triplicate for each donor. A software-assisted Student's $t$ test was performed (SigmaStat v.2.0, Jandel Scientific, USA) and $p<0.05$ was taken as the minimal level of statistical significance.

\section{Results}

\subsection{Expression of PTHrP splicing products in osteo- and adipo- differentiating MSC}

RNA samples isolated at either day 7, 14, 21 or 28 from the three MSC preparations cultured with or without differentiation inducers, were subjected to reverse transcription and the cDNA obtained amplified in the presence of the specific primers.

In a first set of experiments the expression of $3^{\prime}$ end splicing variants, coding for the three PTHrP isoforms of 139, 141 and 173 amino acids, was checked. As shown in Fig. 1A, the splicing isoform encoding for PTHrP 1-139 appeared to be selectively expressed by undifferentiated cells. On the other hand, when the expression of 1-173 splicing isoform was analyzed (Fig. 1B), a positive signal was visualized in all samples, derived from both undifferentiated, osteoand adipo-induced MSC. These results suggest that expression of 1-139 splicing variant might be considered a stemness marker for MSC, whereas the 1-173 splicing isoform appears to be the predominant variant expressed in the model systems under study. In addition, as shown in the histograms, the expression levels of PTHrP 1-173 in all preparations from adipo-differentiating cells were always lower than those from undifferentiated cells, thereby indicating that PTHrP 1-173 down-regulation might be considered an adipo-differentiation specific feature. Conversely, we observed a higher level of expression of this transcript in osteo-differentiating $v s$. undifferentiated cells, suggesting that the up-regulation of this $3^{\prime}$ end splicing isoform might be considered as a novel molecular marker of osteo-induction. No band of $145 \mathrm{bp}$, indicative of the presence of the splicing variants encoding for PTHrP 1-141 isoform, could be observed after amplification of all cDNA preparations (data not shown), indicating that this splicing event is not occurring in MSC both cultured in stem conditions and addressed to differentiation toward the adipo- and osteo-genic lineage.

In a second set of experiments, the expression of the transcript isoforms that are produced from either of the three promoters of PTHrP gene, i.e. P1, P2 and P3 in undifferentiated and differentiation-induced cells, were analyzed. Concerning the isoform produced starting from P1 promoter, it is known that the amplification with specific primers gives rise to different bands depending on the exons enclosed in the transcript. In fact, a band of 


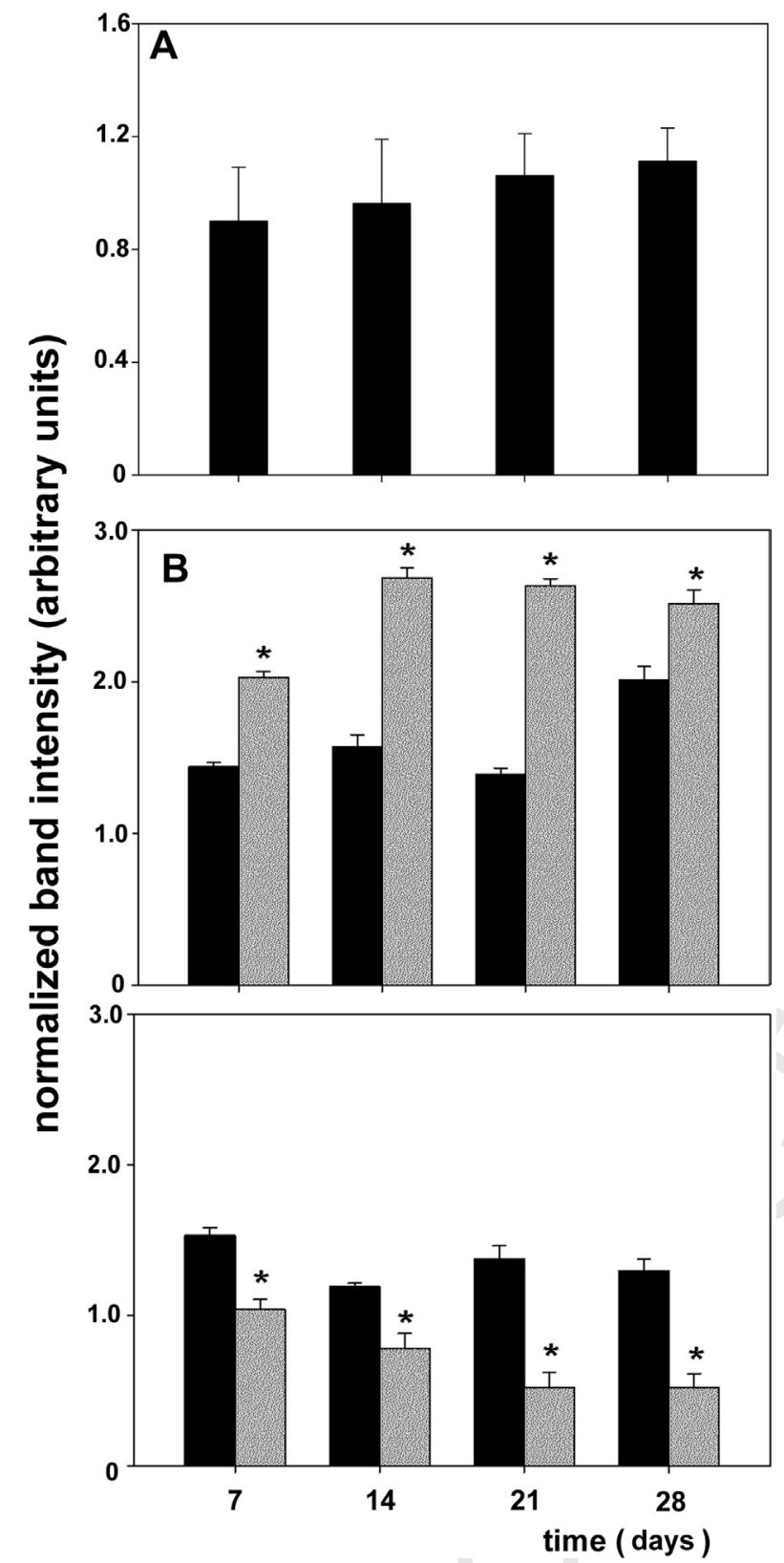

Fig. 1. Expression levels of PTHrP $3^{\prime}$ end mRNA splicing isoforms for 1-139 and 1-173 protein variants in undifferentiated and differentiating MSC. (A) Histogram showing the time-dependent modulation of the expression levels of PTHrP 1-139 mRNA isoform. The histogram reports a prototypic example of the normalized band intensities for undifferentiated MSC, since no amplification signal was observed in preparations from both osteo- and adipo-differentiating MSC. (B) Histograms showing the timedependent modulation of the expression levels of PTHrP 1-173 mRNA isoform in cDNA preparations from undifferentiated (black boxes) osteo- (gray boxes, top) and adipo-differentiating MSC (gray boxes, bottom) at day 7, 14, 21 and 28 from plating. Data are presented as mean \pm s.e.m. of triplicate experiments. ${ }^{*} p<0.05$.

739 bp is obtained when the transcript contains exons I-II-III, whereas bands of 520 and 406 bp are obtained when the transcript contains exons I and III, or exon I only, respectively [25]. As shown in Fig. 2A, when the utilization of P1 was examined, a band of 520 bp was found, indicative of a transcript obtained with the exclusion of exon II, in all cDNA preparations from undifferentiated and differentiating MSC, thus indicating that this variant represents the only $5^{\prime}$ end splicing isoform synthesized from the P1 start site.

Concerning the $\mathrm{P} 2$ promoter-originating isoform, no band of $468 \mathrm{bp}$ indicative of the transcript produced from this promoter
Days: ${ }^{7}+{ }^{14}+-^{21}+-^{28}+8701-\mathrm{BC}$

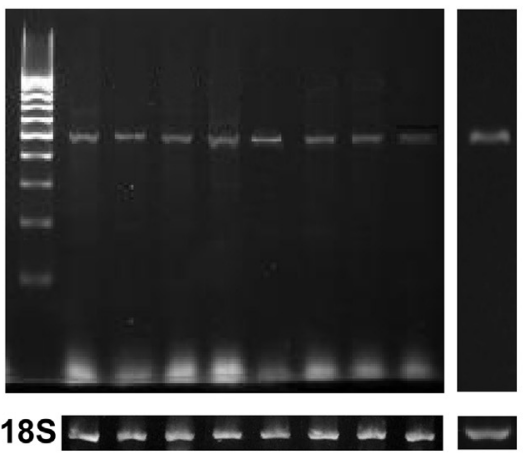

A

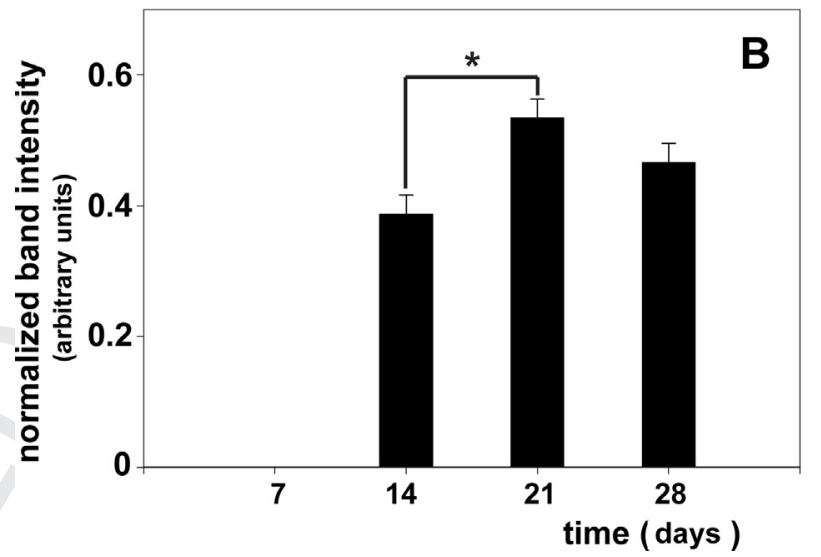

Fig. 2. Expression levels of PTHrP $5^{\prime}$ end mRNA splicing isoforms produced starting from P1 and P3 promoters in undifferentiated and differentiating MSC. A) PCR amplification of the cDNA fragment of the isoform produced starting from P1 promoter from preparations of undifferentiated $(-)$ and osteo-differentiating (+) MSC at day 7 , 14, 21 and 28 from plating, and 8701-BC cells as a positive control, showing the presence of the sole band of $520 \mathrm{bp}$. Below, the amplification of 18S CDNA is shown. The electrophoretic marker in the first lane is 100 bp ladder (Invitrogen). A similar result was obtained when cDNA preparations from adipo-differentiating MSC were utilized (data not shown). Agarose gel 2\%, Red Gel staining. The data presented are representative of 1 donor out of 3. B) Histogram showing the time-dependent modulation of the expression levels of the PTHrP mRNA isoform produced starting from P3 promoter. The histogram reports a prototypic example of the normalized band intensities for undifferentiated MSC, since no amplification signal was observed in preparations from both osteo- and adipo-differentiating MSC. Data are presented as mean \pm s.e.m. of triplicate experiments. ${ }^{*} p<0.05$.

could be observed (data not shown), thereby indicating that P2 is a transcriptionally-inactive start site in MSC under all chosen experimental conditions. On the other hand, after amplification with the specific primers for the P3-derived 5 ' end splicing variant a positive signal could be observed only in cDNA preparations obtained from undifferentiated MSC cultured for at least 2 weeks, with a maximum peak of expression at day 21 from plating (Fig. 2B). The results obtained indicate that the P1 promoter-related transcript is the predominant form synthesized by undifferentiated and differentiating MSC, and suggest that the expression of the P3derived isoform might be considered a stemness marker for MSC.

Since undifferentiated MSC appeared to utilize both the P1 and P3 promoters as transcriptional start sites, in order to evaluate whether one of the two promoters might be more prominently committed for PTHrP transcription the efficiency of PCR amplification of the isoform-specific cDNA fragments was examined. The results obtained indicate that the transcript derived from P1 promoter was more expressed than that from P3, because the correspondent band was already detectable after 35 cycles of amplification, while that from P3 needed more than 40 cycles, following normalization of the data with amplification of 18S cDNA. 

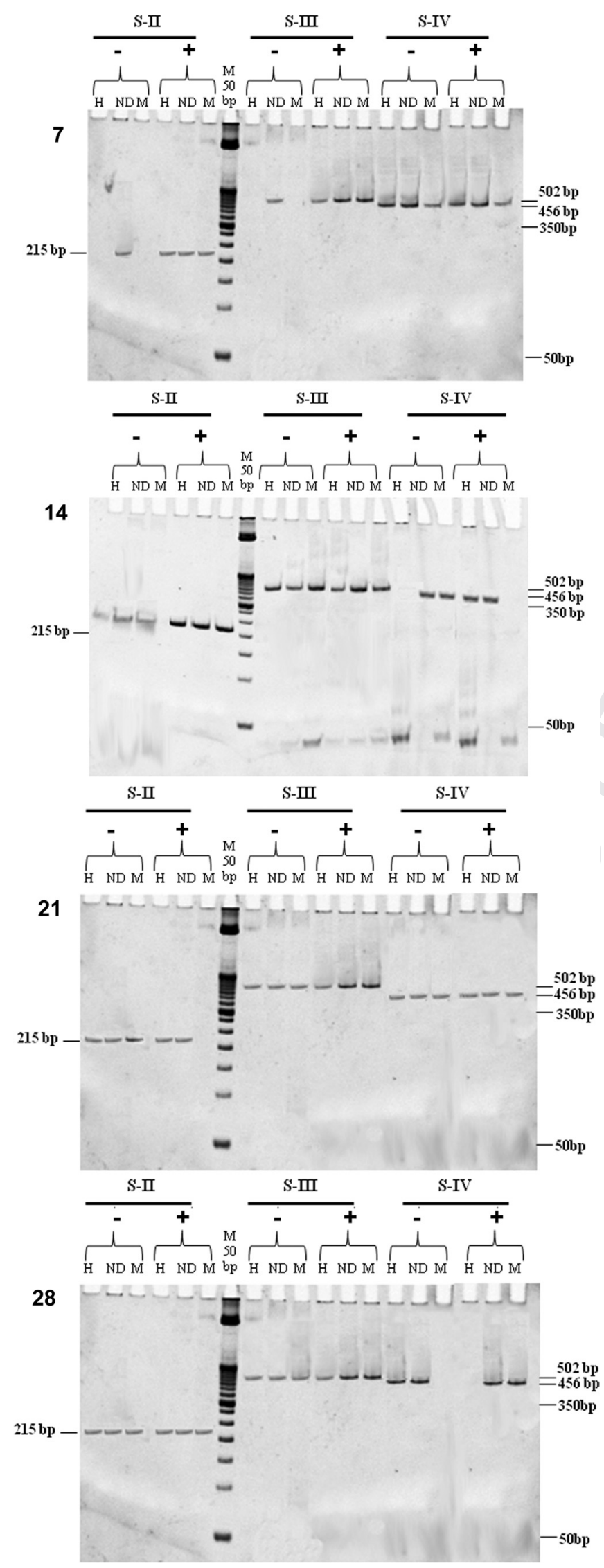

Fig. 3. Time course of P2 promoter methylation in undifferentiated and osteodifferentiating MSC. Methylation analysis of S-II, S-III and S-IV sites of PTHrP P2

\subsection{Methylation state of PTHrP P2 and P3 promoters in undifferentiated and osteo-differentiating MSC}

In light of the obtained data on PTHrP isoform expression showing no $\mathrm{P} 2$ promoter-derived isoform in all preparations tested, as well as the expression of the $\mathrm{P} 3$ promoter-derived transcript isoform only in cDNA preparations from undifferentiated MSC, we therefore examined the methylation status of some internal sites of CpG island of P2 and P3 promoters, in order to check if the promoters were hypermethylated and therefore the expression of the splicing variants silenced, or, in the absence of hypermethylation, whether the presence of insulators could be supposed. Only undifferentiated vs. osteo-differentiating MSC cultures were taken as a prototypic model systems for this part of the study.

MSRE-PCR was performed on genomic DNA isolated at day 7, 14, 21 and 28 from MSC cultured with and without osteogenic differentiation medium. Before PCR amplification, the DNA was pretreated with the methylation-sensitive endonucleases HpaII and MspI, that recognize the same sequence $5^{\prime}$-CCGG- $3^{\prime}$ and cleave the $\mathrm{C}-\mathrm{C}$ bond, but respond in a different way to the state of methylation of C. In particular, the cleavage of DNA by HpalI is prevented by the presence of a 5-methyl group at the internal $C$ residue of the sequence CCGG, whereas MspI cleaves DNA irrespective of the presence of a methyl group except when all the four $C$ of the double strand site are methylated [36]. Therefore, the sites unavailable for cutting by both enzymes were considered as "strongly methylated or hypermethylated", whereas those available for cutting by both MSREs as "unmethylated". The condition in which only one MSRE cleaved the DNA was associated to a "partially methylated" site. The subsequent PCR gives an amplified product only when the MSRE is unable to cut DNA, leaving the template intact and suitable for reaction.

Three sites located within the CpG island of PTHrP P2 promoter, i.e. S-II, S-III and S-IV, were submitted to amplification in the presence of the specific primers. In preparations from MSC at day 7 (Fig. 3A), the 215 and 502 bp amplification product for S-II and S-III, respectively, were visualized after electrophoresis in a polyacrylamide gel only in the undigested DNA sample, while no bands were present in digested DNA preparations, thus demonstrating that S-II and S-III sites were unmethylated. Regarding S-IV site, a $456 \mathrm{bp}$ band was observed in both undigested and digested DNA samples, thereby giving evidence of the strong methylation of this site inhibiting the activity of both MSREs. Conversely, in osteodifferentiating MSC at day 7, S-II, S-III and S-IV sites of the P2 $\mathrm{CpG}$ island were heavily methylated, as shown by the presence of the amplification bands in both undigested and digested DNA preparations. In undifferentiated and osteo-differentiating MSC at day 14 (Fig. 3B), S-II and S-III sites of CpG island were strongly methylated, and S-IV site was partially methylated, as shown by the amplification product originating from the preparation of genomic DNA from undifferentiated MSC restricted with MspI, and from differentiating MSC restricted with HpaII. When S-II and S-III sites were analyzed in samples isolated at day 21 (Fig. 3C), they appeared to be strongly methylated in undifferentiated cells, as demonstrated by the presence of all amplification bands, whereas displaying a partial methylation and a strong methylation state, respectively, in osteo-differentiating cells owing to the presence of

promoter in undifferentiated (-) and osteo-induced $(+)$ MSC at day 7, 14, 21 and 28 from plating. $\mathrm{ND}=$ undigested DNA, $\mathrm{H}=$ DNA digested with HpaII, $\mathrm{M}=$ DNA digested with MspI. The electrophoretic marker in lane 7 is the 50 bp ladder with 375 bp as "flag fragment" (Invitrogen). Twelve percent acrylamide-bisacrylamide (29:1 ratio) gel electrophoresis, SYBR safe staining. The data presented are representative of 1 donor out of 3 . 
215 bp products in both the undigested and the HpaII-digested sample (for S-II site), and of all three 502 bp bands (for the S-III site). S-IV site was shown to be strongly methylated in all the examined preparations and differentiating MSC at day 28 (Fig. 3D), were proven to have strongly methylated S-II and S-III sites of CpG island, since the amplification bands could be observed in both the undigested and digested DNA preparations. S-IV site was partially methylated, since in undifferentiated MSC the amplification product was found in the preparation of genomic DNA restricted with HpaII, while in differentiated MSC in that restricted with MspI.

For P3 promoter, the results of the amplification of genomic DNA from undifferentiated and osteo-differentiated cells at the end of each of the four weeks are shown in Fig. 4. In agreement with gene expression data, preparations from MSC at day 7, both undifferentiated and differentiating, exhibited a band of about $370 \mathrm{bp}$ both in the undigested DNA sample and in that digested with Hpall and $M s p I$, thereby indicating that $\mathrm{CpG}$ sites were methylated. The same electrophoretic pattern was shown by preparations of differentiating MSC at days 14 and 28, whereas the CpG sites at day 21 appeared to be partially methylated. In the undifferentiated cells, the sites under study were not methylated at day 21 and 28, while a hypermethylation of C was observed at day 14 in all MSC preparations tested, this being the only result in disagreement with gene expression data.

\subsection{Intracellular accumulation and extracellular secretion of PTHrP protein product by osteo- and adipo-differentiating MSC} PTHrP isoform expression as a putative molecular signature for MSC stemness/differentiation state, the levels of intracellular accumulation and extracellular secretion of the protein product of PTHrP were studied, to check whether reliable differences could be found between undifferentiated and differentiating cells.

Concerning intracellular accumulation, the comparative analysis of the MICE index in undifferentiated and osteo-differentiating MSC suggested that the accumulation of the PTHrP protein product during the four weeks of treatment with inducers of differentiation was always lower than that found in undifferentiated cells (Fig. 5A). On the other hand, when the same cells were induced toward adipogenic differentiation, the intracellular accumulation of the
In a further set of assays, to complement the data on selected protein did not differ significantly from that of the sample (data not shown).

Extracellular secretion of PTHrP protein product was studied by dot-blot assay on MSC conditioned media. The data obtained showed that in the media of osteo-induced MSC the secreted amounts of PTHrP were always higher than in those harvested from untreated cultures, except for the very early step of differentiation, i.e. day 7 (Fig. 5B). Also in this case, when the same cells were induced toward adipogenic differentiation, the extracellular amount of the secreted protein did not differ significantly to the (data not shown).

\section{Discussion}

PTHrP is a widely-distributed polyprotein coded by a complex transcriptional unit, involved in several tissue-specific differentiation events in which a varying splicing pattern expression of PTHrP mRNA has been reported. For example, in normal human amnion the P2 promoter was proven to be preferentially used and PTHrP 1139 to result the major PTHrP mRNA expressed [37]. In another study on 39 cases of tumors classified as infiltrating ductal carcinoma of the breast, renal cortical carcinoma and lung tumor, and 7 samples of normal tissues (renal, lung and thyroid samples), Southby and coworkers demonstrated that all breast tumor, and both normal and tumor renal samples used each of the three $3^{\prime}$ splicing pathways and therefore expressed mRNA specifying each of the three PTHrP isoforms. Furthermore all tissues examined contained mRNA for the 1-141 isoform, and the mRNA for the 1139 isoform was always present, except in one normal lung and in one parathyroid adenoma sample. On the other hand, the production of the 1-173 isoform was more variable. When the use of the PTHrP promoters was investigated, in contrast to the more restricted utilization of P1 and P2 promoters, P3-initiated transcripts were detected in all tissue samples [25]. Also 8701-BC breast cancer cells were proven to utilize different start sites and mRNA splicing patterns for PTHrP transcription when they were cultured onto different substrates such as reconstituted basement membrane (Matrigel) or representative collagen components of the breast tumor stroma [28], or when exposed to different extracellular $\mathrm{Ca}^{2+}$ concentrations and treated with differentiation agents for breast cells [29].

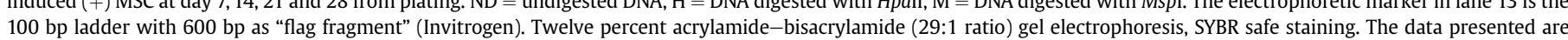
representative of 1 donor out of 3 . 

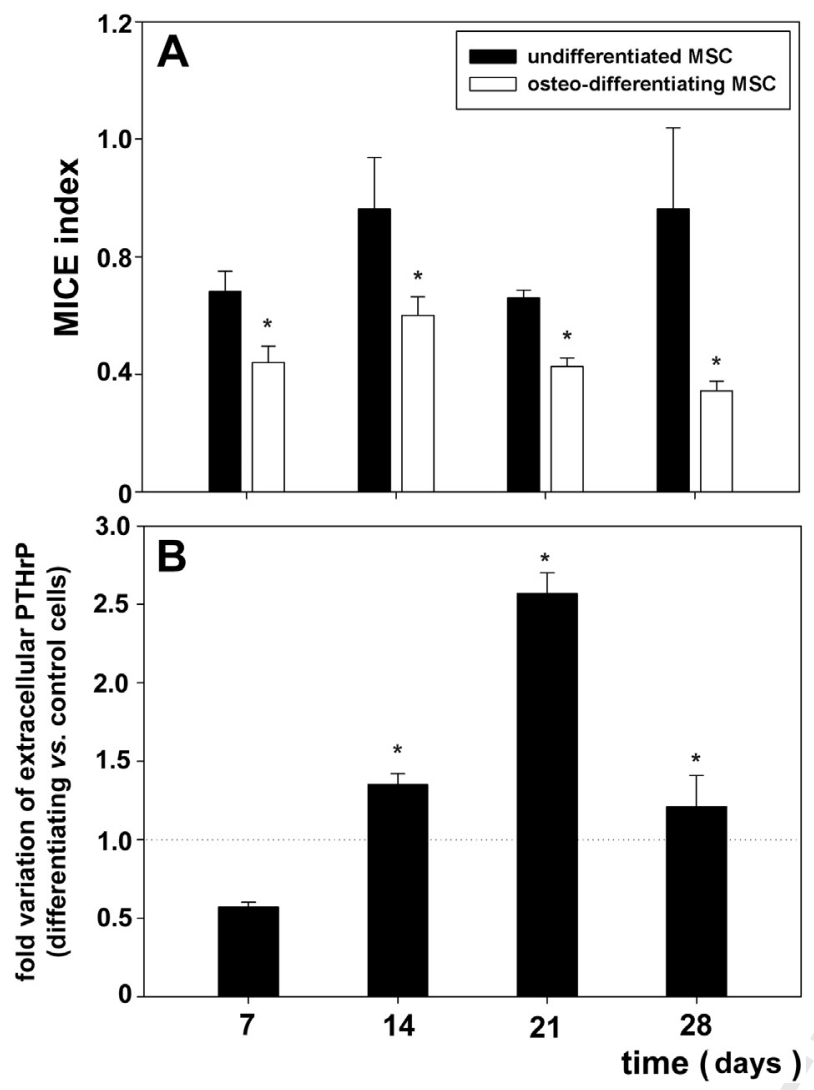

Fig. 5. Intracellular accumulation and extracellular secretion of PTHrP protein product by undifferentiated and osteo-differentiating MSC. Histograms showing the timedependent modulation of $\mathrm{A}$ ) the MICE indexes, indicative of the intracellular levels of PTHrP, and B) the extracellular protein levels of PTHrP from dot blot assay data, in cell preparations and conditioned media from MSC cultured in stem conditions and in the presence of osteogenic differentiation medium for 4 weeks. The histogram in B) indicate the ratio between the amounts secreted by differentiating $v s$. undifferentiated cells. Data are presented as mean \pm s.e.m. of triplicate experiments. ${ }^{*} p<0.05$.

Little is known about the expression and the accumulation of PTHrP transcript isoforms in adult MSC either undifferentiated or differentiating toward osteo- and adipo-genic lineages. The literature data focus, almost exclusively, on the effect of different PTHrP protein domains on the osteo-, chondro- and adipo-genic potential of MSC [e.g. Refs. [37-39]]. Here we report that PTHrP splicing isoforms are expressed in MSC both undifferentiated and differentiating. In particular, on the basis of the data obtained on the three different cell cultures here studied the following panel of expression of PTHrP transcript isoforms in the different experimental conditions can be schematized (Table 3), indicating that from the stemness condition to that of osteo- and adipo-genic differentiated cells, the expression of isoforms becomes increasingly selective, thereby being a potential target for the monitoring of cell stem or committed/differentiating state. Noteworthy, literature data indicate that dexamethasone may down-regulate $\mathrm{PTHrP}$ expression of adult stem cells in a dose-dependent manner [40]. Taking into consideration that the hormone is present in both culture media used for MSC differentiation but in different concentration ( $1 \mu \mathrm{M}$ for adipo-induction, $0.1 \mu \mathrm{M}$ for osteo-induction), it is conceivable that it might be responsible, at least in part, for the decreased PTHrP expression observed in MSC addressed to adipodifferentiation.

Interestingly, preliminary results obtained with dental follicle MSC of wisdom teeth, isolated during routine dental surgery [41], show that these cells use solely the P3 promoter for the expression of splicing variants (Longo, Tobiasch and Luparello, unpubl. data), thereby suggesting that MSC from different tissue sources may be endowed with differing transcriptional programmings. Therefore, caution must be exercised in generalizing the data arising from experiments on MSC depending on their origin, since they should not be considered as a single homogeneous cytotype.

The role of epigenetic modifications in PTHrP expression has been the object of a limited number of literature reports. Using normal and lung squamous carcinoma cells as model systems, the study of the methylation status of $\mathrm{CpG}$ dinucleotides in the $5^{\prime}$ region of the gene showed that in the former case the CpG islands were completely unmethylated, whereas in neoplastic cells, twothirds of the CpG islands were substantially methylated. Reverse transcriptase-PCR analysis showed that this heavy methylation did not prevent expression of any of the three PTHrP gene promoters, highlighting that in the model systems under study methylation was not associated with inhibition of gene activity [20]. Results obtained with a human model of mammary epithelial cell lines differing in tumorigenicity and $\mathrm{PTHrP}$ expression, have suggested that the methylation status of specific $\mathrm{CpG}$ dinucleotides in the P2 promoter is the dominant mechanism involved in silencing of PTHrP expression rather than the overall methylation of the CpG island. Methylation of the PTHrP P2 promoter might represent a potential marker of breast cancer progression and be used to evaluate the metastatic potential of breast tumors [42].

In our study, the lack of P2 promoter-deriving transcription in MSC under all experimental conditions, as well as of P3 promoterderiving transcription in differentiating MSC, prompted to enter more into details through epigenetic analyses. The analysis of the methylation status of the S-II, S-III and S-IV sites in CpG islands of the P2 promoter in osteo-induced MSC from one donor by MSREPCR showed a predominant hypermethylation or partial methylation at these sites. These data, therefore, may be related to those obtained on P2 promoter-derived splicing isoform expression by MSC, which had demonstrated that this transcript was not expressed by cells under every culture condition, and suggest that the $\mathrm{CpG}$ islands contained in the P2 promoter is of prime importance in regulating gene expression, consequently excluding the hypothesis that these sites can accommodate a sequence insulator. Concerning the $\mathrm{CPG}$ site of the $\mathrm{P} 3$ promoter, in agreement with gene expression data, a condition of strong methylation was found

Table 3

Synopsis of $P T H r P$ splicing isoforms expressed in undifferentiated, osteo- and adipo-differentiating MSC.

\begin{tabular}{|c|c|c|c|c|c|c|}
\hline \multirow{3}{*}{$\begin{array}{l}\text { Cells } \\
\text { Undifferentiated MSC }\end{array}$} & \multirow{3}{*}{$\begin{array}{l}\begin{array}{l}\text { Promoter } \\
\text { used }\end{array} \\
\text { P1 } \\
\text { P3 }\end{array}$} & \multirow{3}{*}{$\begin{array}{l}\text { Exons included } \\
\text { at the } 5^{\prime} \text { end } \\
\text { I-III } \\
\text { IV }\end{array}$} & \multirow{3}{*}{$\begin{array}{l}\text { Ever-present } \\
\text { exons } \\
\text { V-VI } \\
\text { V-VI }\end{array}$} & \multicolumn{2}{|c|}{$\begin{array}{l}\text { Exons included at } 3^{\prime} \text { end } \rightarrow \text { size of } \\
\text { isoform protein }\end{array}$} & \multirow{3}{*}{$\begin{array}{l}\text { Resulting mRNA and potential protein } \\
\text { isoforms } \\
\begin{array}{l}\text { Four transcript isoforms, potentially } \\
\text { two protein isoforms }\end{array}\end{array}$} \\
\hline & & & & $\mathrm{VII} \rightarrow 139$ aa & VIII $\rightarrow 173$ aa & \\
\hline & & & & $\mathrm{VII} \rightarrow 139$ aa & VIII $\rightarrow 173$ aa & \\
\hline Osteo-differentiating MSC & $\mathrm{P} 1$ & I-III & $\mathrm{V}-\mathrm{VI}$ & $\begin{array}{l}\text { VII } \rightarrow 139 \text { aa } \\
\text { (early time only) }\end{array}$ & $\mathrm{VIII} \rightarrow 173 \mathrm{aa}$ & $\begin{array}{l}\text { Two transcript isoforms, potentially } \\
\text { two protein isoforms }\end{array}$ \\
\hline Adipo-differentiating MSC & P1 & I-III & $\mathrm{V}-\mathrm{VI}$ & VIII $\rightarrow 173$ aa & & $\begin{array}{l}\text { One transcript isoform, one protein } \\
\text { isoform }\end{array}$ \\
\hline
\end{tabular}


in all four weeks of differentiation, whereas, in the undifferentiated cell counterpart, hypermethylation was present at day 7, whereas demethylation at day 21 and 28 . The only result discordant with the previously-obtained gene expression data is related to the strong methylation of P3 promoter in all samples of undifferentiated MSC at day 14. Although further validation of this result in other MSC preparations might be necessary, if confirmed this means that such hypermethylation is unable to inhibit the expression of the splicing isoform in this stage of the cell culture. Additional studies will be therefore performed to understand the true role of P3 CpG sites, even testing the hypothesis that can accommodate insulator sequences.

In search for molecular markers relevant in the early monitoring of the correct differentiation progress of MSC, we also evaluated whether differences could be found in the rate of intracellular accumulation and/or extracellular secretion of the protein product of PTHrP. The combined data obtained from MICE and dot blot assays have put in evidence that PTHrP intracellular retention is down-regulated in osteo-differentiating MSC which display a higher level of secretion of the protein in the extracellular medium with respect to stem cells, except for the early differentiation stage, i.e. the first week of osteo-induction. When adequately confirmed, these variations of the intracellular and extracellular levels of PTHrP could potentially be enclosed in the list of the available protein signature of osteogenic differentiation.

\section{Conclusion}

In conclusion, the collective data obtained have enlightened some aspects in the complex and tightly-regulated biological process of adipogenic and osteogenic differentiation by adult MSC, also indicating that PTHrP expression and production might be considered as signatures useful for monitoring the correct progress of MSC differentiation. Further investigations will be useful to unravel the molecular processes that regulate the differentiation events of human somatic stem cells, which offer opportunities to get more insight into questions in human biology and can promote the advancement of clinical research helping the development of new therapies and a better evaluation of biosecurity issues in clinical trials.

\section{Acknowledgments}

The work was supported by University of Palermo (R.S. ex60\% and FFR 2013) and Progetto Vigoni 2011 for Claudio Luparello, and FC, BMBF, AdiPaD, 1720X06, Geräteprogramm, Fachhochschulen NRW 2008, HIFF, Fördergesellschaft der Fachhochschule BonnRhein-Sieg and DAAD, PPP, D/07/13409 for Edda Tobiasch.

\section{References}

[1] A. Schaffler, C. Buchler, Concise review: adipose tissue-derived stromal cells basic and clinical implications for novel cell-based therapies, Stem Cells 25 (2007) 818-827.

[2] Z.J. Liu, Y. Zhuge, O.C. Velazquez, Trafficking and differentiation of mesenchymal stem cells, J. Cell. Biochem. 106 (2009) 984-991.

[3] A. Pansky, B. Roitzheim, E. Tobiasch, Differentiation potential of adult human mesenchymal stem cells, Clin. Lab. 53 (2007) 81-84.

[4] B.A. Bunnell, M. Flaat, C. Gagliardi, B. Patel, C. Ripoll, Adipose-derived stem cells: isolation, expansion and differentiation, Methods 45 (2008) 115-120.

[5] C.B. Baer, H. Geiger, Adipose-derived mesenchymal stromal/stem cells: tissue localization, characterization, and heterogeneity, Stem Cells Int. (2012), http:// dx.doi.org/10.1155/2012/812693.

[6] M. Locke, J. Windsor, P.R. Dunbar, Human adipose-derived stem cells: isolation, characterization and applications in surgery, ANZ J. Surg. 79 (2009) 235-244.

[7] Y. Zhang, D. Khan, J. Delling, E. Tobiasch, Mechanisms underlying the osteoand adipo-differentiation of human mesenchymal stem cells, ScientificWorldJournal (2012), http://dx.doi.org/10.1100/2012/793823.
[8] Q.Q. Tang, T.C. Otto, M.D. Lane, Commitment of C3H10T1/2 pluripotent stem cells to the adipocyte lineage, Proc. Natl. Acad. Sci. U. S. A. 101 (2004) 9607-9611.

[9] W. Huang, S. Yang, J. Shao, Y.P. Li, Signaling and transcriptional regulation in osteoblast commitment and differentiation, Front. Biosci. 12 (2007) 3068-3092.

[10] G. Karsenty, Transcriptional of skeletogenesis, Ann. Rev. Genomics Hum. Genet 9 (2008) 183-196.

[11] S. Muruganandan, A.A. Roman, C.J. Sinal, Adipocyte differentiation of bone marrow-derived mesenchymal stem cells: cross talk with the osteoblastogenic program, Cell. Mol. Life Sci. 66 (2009) 236-253.

[12] C. Luparello, W.J. Burtis, F. Raue, M.A. Birch, J.A. Gallagher, Parathyroid hormone-related peptide and 8701-BC breast cancer cell growth and invasion in vitro: evidence for growth-inhibiting and invasion-promoting effects, Mol Cell. Endocrinol. 111 (1995) 225-232.

[13] C. Luparello, M.A. Birch, J.A. Gallagher, W.J. Burtis, Clonal heterogeneity of the growth and invasive response of a human breast carcinoma cell line to parathyroid hormone-related peptide fragments, Carcinogenesis 18 (1997) 23-29.

[14] C. Luparello, R. Romanotto, A. Tipa, R. Sirchia, N. Olmo, I. López de Silanes, J. Turnay, M.A. Lizarbe, A.F. Stewart, Midregion parathyroid hormone-related protein inhibits growth and invasion in vitro and tumorigenesis in vivo of human breast cancer cells, J. Bone Miner. Res. 16 (2001) 2173-2181.

[15] E. Maioli, V. Fortino, C. Torricelli, PTHrP/PTH1R: a complex crosstalk among different signaling pathways, in: C. Luparello (Ed.), Novel Aspects of PTHrP Physiopathology, Nova Sci. Publ., New York, 2007, pp. 143-164.

[16] E.J. Sanders, S. Harvey, Peptide hormones as developmental growth and differentiation factors, Dev. Dyn. 237 (2008) 1537-1552.

[17] C. Luparello, Parathyroid hormone-related-protein (PTHrP): a key regulator of life/death decisions by tumor cells with potential clinical applications, Cancers 3 (2011) 396-407.

[18] Y.J. Kim, H.J. Kim, G.I. Im, PTHrP promotes chondrogenesis and suppresses hypertrophy from both bone marrow-derived and adipose tissue-derived MSCs, Biochem. Biophys. Res. Commun. 373 (2008) 104-108.

[19] S. Weiss, T. Hennig, R. Bock, E. Steck, W. Richter, Impact of growth factors and PTHrP on early and late chondrogenic differentiation of human mesenchymal stem cells, J. Cell. Physiol. 223 (2010) 84-93.

[20] R.H. Ganderton, R.S. Briggs, CpG island methylation and promoter usage in the parathyroid hormone-related protein gene of cultured lung cells, Biochim. Biophys. Acta 1352 (1997) 303-310.

[21] R.H. Ganderton, R.S. Briggs, Increased upstream methylation has no influence on the overexpression of the parathyroid hormone-related protein gene in squamous cell carcinoma of the lung, Eur. J. Cancer 36 (2000) 2128-2136.

[22] M.T. Gillespie, T.J. Martin, The parathyroid hormone-related protein gene and its expression, Mol. Cell. Endocrinol. 100 (1994) 143-147.

[23] J.F. Whitfield, Parathyroid hormone-related protein (PTHrP): an ancient string of cytokines with many known and still unknown functions, in: C. Luparello (Ed.), Novel Aspects of PTHrP Physiopathology, Nova Sci. Publ., New York, 2007, pp. 1-25

[24] C. Luparello, Midregion PTHrP and human breast cancer cells, ScientificWorldJournal 10 (2010) 1016-1028.

[25] J. Southby, L.M. O’Keeffe, T.J. Martin, M.T. Gillespie, Alternative promoter usage and mRNA splicing pathways for parathyroid hormone-related protein in normal tissues and tumours, Br. J. Cancer 72 (1995) 702-707.

[26] G. Wu, M. Iwamura, P.A. di Sant'Agnese, L.J. Deftos, A.T. Cockett, S. Gershagen, Characterization of the cell-specific expression of parathyroid hormonerelated protein in normal and neoplastic prostate tissue, Urology 51 (1998) $110-120$.

[27] Z. Bouizar, F. Spyratos, M.C. De Vernejoul, The parathyroid hormone-related protein (PTHrP) gene: use of downstream TATA promotor and PTHrP 1-139 coding pathways in primary breast cancers vary with the occurrence of bone metastasis, J. Bone Miner. Res. 14 (1999) 406-414.

[28] C. Luparello, T. Schilling, R. Cirincione, I. Pucci-Minafra, Extracellular matrix regulation of $\mathrm{PTHrP}$ and $\mathrm{PTH} / \mathrm{PTHrP}$ receptor in a human breast cancer cel line, FEBS Lett. 463 (1999) 265-269.

[29] C. Luparello, F. Santamaria, T. Schilling, Regulation of PTHrP and PTH/PTHrP receptor by extracellular $\mathrm{Ca}^{2+}$ concentration and hormones in the breast cancer cell line 8701-BC, Biol. Chem. 381 (2000) 303-308.

[30] R.S. Sellers, A.I. Luchin, V. Richard, R.M. Brena, D. Lima, T.J. Rosol, Alternative splicing of parathyroid hormone-related protein mRNA: expression and stability, J. Mol. Endocrinol. 33 (2004) 227-241.

[31] N. Zippel, C.A. Limbach, N. Ratajski, C. Urban, C. Luparello, A Pansky, M.U. Kassack, E. Tobiasch, Purinergic receptors influence the differentiation of human mesenchymal stem cells, Stem Cells Dev. 21 (2012) 884-900.

32] C. Luparello, F. David, G. Campisi, R. Sirchia, T47-D cells and type V collagen: a model for the study of apoptotic gene expression by breast cancer cells, Biol. Chem. 384 (2003) 965-975.

[33] F. Caradonna, G. Barbata, G. Sciandrello, Genomewide hypomethylation and PTHrP gene hypermethylation as a model for the prediction of cancer risk in rheumatoid arthritis, in: C. Luparello (Ed.), Novel Aspects of PTHrP Physiopathology, Nova Sci. Publ., New York, 2007, pp. 305-319.

[34] S.M. De la Monte, N. Ganju, J.R. Wands, Microtiter immunocytochemical ELISA assay, BioTechniques 26 (1999) 1073-1078.

[35] R. Barbieri, G. Duro, M.A. Costa, V. Izzo, Simple and inexpensive dot-blot apparatus, Anal. Biochem. 216 (1994) 461-462.

[36] S. Tardy-Planechaud, J. Fujimoto, S.S. Lin, L.C. Sowers, Solid phase synthesis and restriction endonuclease cleavage of oligodeoxynucleotides containing 5(hydroxymethyl)-cytosine, Nucleic Acids Res. 25 (1997) 553-558. 
[37] D.W. Brandt, M. Bruns, D.E. Bruns, J.E. Ferguson II, D.W. Burton, L.J. Deftos, The parathyroid hormone-related protein (PTHrP) gene preferentially utilizes a GC-rich promoter and the pthrp 1-139 coding pathway in normal human amnion, Biochem. Biophys. Res. Commun. 189 (1992) 938-943.

[38] A. Casado-Diaz, R. Santiago Mora, J.M. Quesada, The N- and C-terminal domains of parathyroid hormone-related protein affect differently the osteogenic and adipogenic potential of human mesenchymal stem cells, Exp. Mol. Med. 42 (2010) 87-98.

[39] J.M. Lee, G.I. Im, PTHrP isoforms have differing effect on chondrogenic differentiation and hypertrophy of mesenchymal stem cells, Biochem. Biophys. Res. Commun. 421 (2012) 819-824.
[40] M. Ahlström, M. Pekkinen, C. Lamberg-Allardt, Dexamethasone downregulates the expression of parathyroid hormone-related protein $(\mathrm{PTHrP})$ in mesenchymal stem cells, Steroids 74 (2009) 277-282.

[41] E.M. Haddouti, M. Skroch, N. Zippel, C. Müller, B. Birova, A. Pansky, C. Kleinfeld, M. Winter, E. Tobiasch, Human dental follicle precursor cells of wisdom teeth: isolation and differentiation towards osteoblasts for implants with and without scaffolds, Materialwiss. Werkstofftech. 40 (2009) 732-737.

[42] J. Tost, H. Hamzaoui, F. Busato, A. Neyret, S. Mourah, J.M. Dupont, Z. Bouizar, Methylation of specific $\mathrm{CpG}$ sites in the $\mathrm{P} 2$ promoter of parathyroid hormonerelated protein determines the invasive potential of breast cancer cell lines, Epigenetics 6 (2011) 1035-1046. 